\title{
X-ray Detection With Multi-anode Sawtooth Silicon Drift Detectors ${ }^{1}$
}

\author{
J. Šonský², J. Huizenga ${ }^{2}$, R.W. Hollander ${ }^{2}$, Member, IEEE C.W.E. van Eijk ${ }^{2}$, Member, IEEE \\ and P.M. Sarro ${ }^{3}$ \\ ${ }^{2}$ Radiation Technology Group, Interfaculty Reactor Institute, Delft University of Technology, Mekelweg 15, 2629 JB Delft, \\ The Netherlands \\ ${ }^{3}$ Delft Institute of Microelectronics and Submicron Technology, Delft University of Technology, Mekelweg 4, 2628 CD Delft, \\ The Netherlands
}

\begin{abstract}
We present results of room temperature and lowtemperature X-ray measurements with $500 \mu \mathrm{m}$ anode pitch Multi-anode Linear and Sawtooth Silicon Drift Detectors. An analysis of the influence of split events due to the lateral spread of the drifting electron cloud, electronic noise and the general spectroscopic performance of the detectors is given. An energy resolution of $450 \mathrm{eV} \mathrm{FWHM}$ was determined for the $5.89 \mathrm{keV}$ line of ${ }^{55} \mathrm{Fe}$ at $233 \mathrm{~K}$. Split events are completely eliminated.
\end{abstract}

\section{INTRODUCTION}

Position sensitive detection of low energy X-rays can be realized by means of a Multi-anode Linear Silicon Drift Detector (MLSDD). One-dimensional position information in the lateral direction (perpendicular to the drift direction) is obtained from the $\mathrm{n}^{+}$anode pixel signals. We intend to develop a $250 \mu \mathrm{m}$ anode pitch MLSDD with a very good energy resolution for detection of X-rays from $180 \mathrm{eV}\left(\mathrm{K}_{\alpha}\right.$ line of boron) to $10 \mathrm{keV}$. A key feature of the Silicon Drift Detectors (SDDs) is its very small anode capacitance ( $\sim 100 \mathrm{fF})$ allowing an ultimately good energy resolution. To fully exploit its feature, an integration of the front-end JFET of pre-amplifier is necessary. An energy resolution of $137 \mathrm{eV}$ FWHM (7.9 rms electrons) at the $\mathrm{Mn}-\mathrm{K}_{\alpha}$ line of the ${ }^{55} \mathrm{Fe}$ spectrum was measured with such configuration at a temperature of $220 \mathrm{~K}$ [1]. Also energy losses at the entrance window can be made small enough even for very low-energy X-rays [2]. Therefore, $\mathrm{X}$-rays at the low-energy side of the required energy range can be sufficiently detected.

Circular SDDs are usually used for X-ray spectroscopy so far. In a circular SDD, the drift field sweeps the signal electron cloud radially inwards to one central anode and energy resolution is determined only by the intrinsic charge generation statistics and the electronic noise. In the case of an MLSDD, diffusion broadens the signal electron cloud laterally during its drift towards the array of anode pixels [3]. Consequently a position dependent loss of charge to adjacent anode pixels is observed. In pulse height spectrum measurements per pixel, the incomplete charge collection (or split events) generally results in a low-energy tailing of the full-energy gaussian peaks. To illustrate the influence of diffusion, we have plotted in Fig. 1 the calculated spectrum of a $6 \mathrm{keV}$ monochromatic $\mathrm{X}$-ray source collected at one anode pixel of MLSDDs with the

\footnotetext{
${ }^{1}$ This research has been supported by the Netherlands Technology Foundation (STW).
}

anode pitch as a parameter [4]. Ballistic deficit, edge effects, and doping inhomogeneities of silicon wafers are other, less important, sources of incomplete charge collection in large MLSDDs.

Based on the character of the split events, several parameters can be introduced to quantify the influence of split events on the quality of MLSDD X-ray spectra. Peak-to-background ratio $\left(\mathrm{R}_{\text {count }}\right)$ is the most general one. We can define $\mathrm{R}_{\text {count }}$ as a ratio between the full-energy peak efficiency and the split events (or background) efficiency. The total observed energy resolution expressed, if possible, as the full-width at half-maximum $\left(\mathrm{FWHM}_{t o t a l}\right)$ can be written as

$$
F W H M_{\text {total }}^{2}=F W H M_{i n t}^{2}+F W H M_{e l}^{2}+F W H M_{s p l i t}^{2}
$$

where FWHM $_{\text {int }}$ is the intrinsic resolution of the detector, $\mathrm{FWHM}_{e l}$ is the resolution due to the electronic noise $\left(F W H M_{\mathrm{el}}=2.35 e E N C\right.$, where $e(\mathrm{in} \mathrm{eV})$ is the mean energy required to generate an electron-hole pair and $\mathrm{ENC}$ is the electronic noise expressed in rms electrons), and $\mathrm{FWHM}_{\text {split }}$ is the energy resolution due to the split events and follows from experiment. For simplification, we also define $F W H M^{2}=F W H M_{i n t}^{2}+F W H M_{e l}^{2}$. Obviously the $\mathrm{FWHM}_{\text {total }}$ can be used only if the full-energy events dominate over the split events (see Fig. 1). If the split events result in a steady continuous background (i.e. the edge effects),

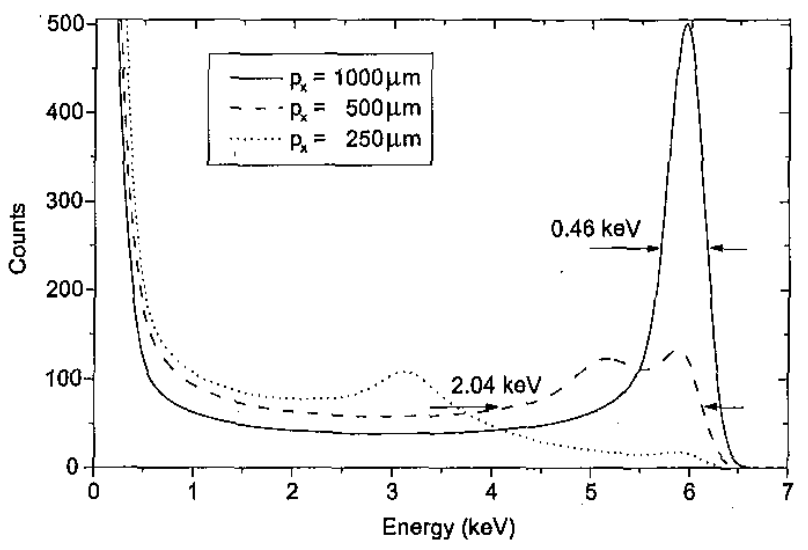

Figure 1: Calculated pulse height spectra of a $6 \mathrm{keV}$ monochromatic $\mathrm{X}$-ray source collected at one anode pixel of a MLSDD with a different anode pitch $p_{x}$ at room temperature $(T=298 \mathrm{~K})$. We assumed a uniform irradiation of the entire active area. The FWHM $_{t o t a l}$ is indicated for $\mathrm{p}_{x}=500$ and $1000 \mu \mathrm{m}$. 
it is practical to use a peak-to-valley ratio $\left(\mathrm{R}_{\text {peak }}\right) . \mathrm{R}_{\text {peak }}$ is defined as a ratio between the height of the full-energy peak and the average height of the background. The disadvantage of $\mathrm{R}_{\text {peak }}$ is its dependency on the electronic noise and therefore a straight comparison of spectra recorded with different detectors is not always possible.

To avoid the problem of split events in an MLSDD caused by lateral spread of the electron cloud due to diffusion, potential gutters confining the drifting electron cloud in the lateral direction are necessary. This can be realized by means of sawtooth shaped $\mathrm{p}^{+}$strips, the sawtooth concept [5]. The depth of induced potential gutters is determined by the sawtooth period $\mathrm{p}_{x}$ (= anode pitch), strip pitch $\mathrm{p}_{y}$, sawtooth angle $\alpha$, and operating drift field $[5,6]$. We call this new drift detector Multi-anode Sawtooth Silicon Drift Detector (MSSDD). In this paper, we present a comparison of X-ray spectra obtained with a $500 \mu \mathrm{m}$ anode pitch MSSDD $\left(\alpha \geq 30^{\circ}\right)$ and a MLSDD $(\alpha$ $=0^{\circ}$ ) at room and low temperatures. An extensive discussion of the origins of split events, the electronic noise, and the performance of the detector is given.

\section{Detector Design, Housing, And ELECTRONICS}

MSSDDs with a total active area of $2.5 \times 1.3 \mathrm{~cm}^{2}$ have been fabricated on 4-inch wafers of Czochralski type silicon with a resistivity of $2-10 \mathrm{k} \Omega \mathrm{cm}$ and a thickness of $500 \mu \mathrm{m}$. The detector is a bi-directional MSSDD with a maximal drift distance of $1.25 \mathrm{~cm}$ in both directions (see Fig. 2a). The anode pitch $\left(\mathrm{p}_{x}\right)$ is $500 \mu \mathrm{m}$ and the strip pitch $\left(\mathrm{p}_{y}\right)$ is $200 \mu \mathrm{m}$. The test detector is divided in 4 sections with sawtooth angle $\alpha=$ $0^{\circ}, 30^{\circ}, 45^{\circ}$, and $60^{\circ}$. The detailed description of the detector design can be found in $[6,7]$.

The detectors were mounted on ceramic boards which allow cooling down to $\sim 210 \mathrm{~K}$. The front-end JFETs of the preamplifiers are mounted close to the read-out anode pixels (see Fig. 2b) to minimize the stray capacitance (the length of the wire connecting an anode pixcl with a gate of the JFET is $\sim 2 \mathrm{~mm}$ and we expect a stray capacitance $\mathrm{C}_{s t} \sim 1.5 \mathrm{pF}$ ). The rest of the preamplifier is placed on the ceramic board. The low-noise housing for room temperature measurements included a Peltier cooler for stabilization of the detector temperature. A vacuum set-up with liquid nitrogen cooling was used to carry out the low temperature measurements. The signals were processed by an ORTEC 672 spectroscopy amplifier with a semi-gaussian filter and fed into an ORTEC ADI 14. We have used ${ }^{241} \mathrm{Am}$ and ${ }^{55} \mathrm{Fe}$ radioactive sources for X-ray spectroscopy. For measurement of the drift time, a pulsed laser $(\lambda=675 \mathrm{~nm}$ ) was used to generate signal electrons. The optical fiber with a focusing lens is placed on an $\mathrm{XY}$ micrometric stage allowing generation of the signal electrons along the active area of the detector. The leakage

(a)

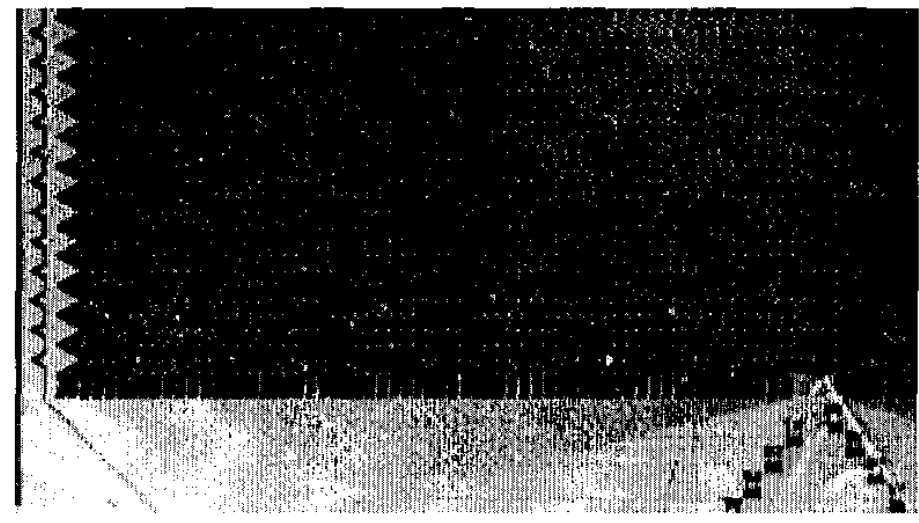

(b)

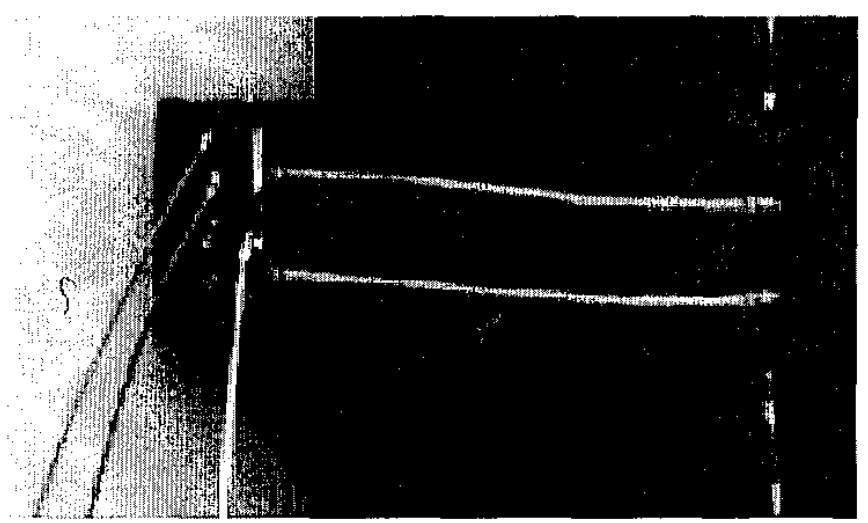

Figure 2: Digitized photograph showing the design of the back-side (p-side) of MSSDD (a) and JFETs connected to anode pixels (b). 
currents were measured with a Keithley model 6517.

In order to obtain a very good energy resolution we have used a low-noise preamplifier [8], where the feedback capacitor is discharged by a slightly forward biased gate-to-source junction of a p-channel JFET. The commercially available p-channel JFETs have a very high input capacitance $(\sim$ a few $\mathrm{pF}$ ) and therefore we have designed and processed a low-noise p-channel JFETs (PJF100) with a gate width of $100 \mu \mathrm{m}$ and a gate length of $1 \mu \mathrm{m}$. The JFET is top-gate driven and at operating point, the input capacitance $\left(C_{i}\right)$ is about $0.5 \mathrm{pF}$, the cutoff frequency $f_{t}=g_{m} /\left(2 \pi C_{i}\right)$ is about $1 \mathrm{GHz}$ and the $1 / \mathrm{f}$ noise parameter $P_{f}=A_{f} C_{i}$ is about $8 \times 10^{-25} \mathrm{~J}$, where $\mathrm{A}_{f}$ is the physical spectral density at $f=1 \mathrm{~Hz}$ of the $1 / \mathrm{f}$ noise. The capacitance $\left(\mathrm{C}_{f b}\right)$ of the feedback capacitor is $50 \mathrm{fF}$. The JFETs together with the feedback capacitors are fabricated using the DIMES-03 process [9].

\section{EXPERIMENTAL RESULTS}

\section{A. Biasing and Drift Time Measurement}

Concerning the biasing of the MSSDD, we have applied on the front-side (n-side) a negative potential which is decreasing in uniform steps from the outermost $\mathrm{p}^{+}$strip to the first $\mathrm{p}^{+}$ strip (closest to the anode). The negative voltage on the backside ( $\mathrm{p}$-side) is the same (drift region) up to the focusing region below the array of anode pixels. In this region an increasing negative potential deflects the drifting electrons from the middle of the wafer towards the anode pixels. Moreover a potential applied on the $\mathrm{p}^{+}$guard strip underlying the $\mathrm{n}^{+}$guard (on the $n$-side) prevents collection of electrons generated outside the active area on the anode pixels. The applied drift field was $\sim 200$ $\mathrm{V} / \mathrm{cm}$. To realize this potential distribution, a simple external resistor chain is used. The latter provides an individual bias of only four $\mathrm{p}^{+}$strips (two on both sides) in the drift region and two $\mathrm{p}^{+}$strips, the $\mathrm{p}^{+}$focusing and the $\mathrm{p}^{+}$guard strip, in the focusing region. The other $\mathrm{p}^{+}$strips are biased by means of two chains of implanted resistors of $200 \mathrm{k} \Omega$. If the biasing is done in this manner, the relatively small current flowing in the internal resistor chain can be more easily affected by the leakage currents collected at the $\mathrm{p}^{+}$strips and consequently the

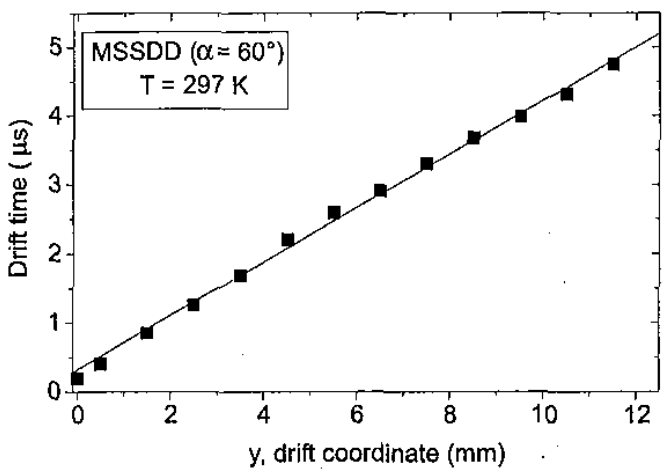

Figure 3: Drift time as a function of drift coordinate (distance from the array of anode pixels). The straight line shows the linear fit. resulting potential along the detector would not be linear [10]. To verify the linearity of the biasing, we have measured the drift time as a function of the drift coordinate (see Fig. 3). A good fit by a straight regression line is observed.

\section{B. Leakage Current}

The leakage current collected at one anode pixel was measured as a function of temperature for different sawtooth angles $\alpha$ (see Fig. 4). The measured leakage current per $\mathrm{cm}^{2}$ is higher by at least a factor of 3 as compared with a circular SDD produced with the same technology [11]. In general, the biasing of the large SDD with a linear geometry is more complicated and the effects, like high electric fields at the sharp corners of $\mathrm{p}^{+}$strips and the leakage current generated at the detector edges, have to be treated carefully. Despite these complications, it should be possible to achieve results similar to the ones measured with circular SDD. The leakage currents presented in Fig. 4 were measured on one detector only. Therefore general conclusions can not be drawn.

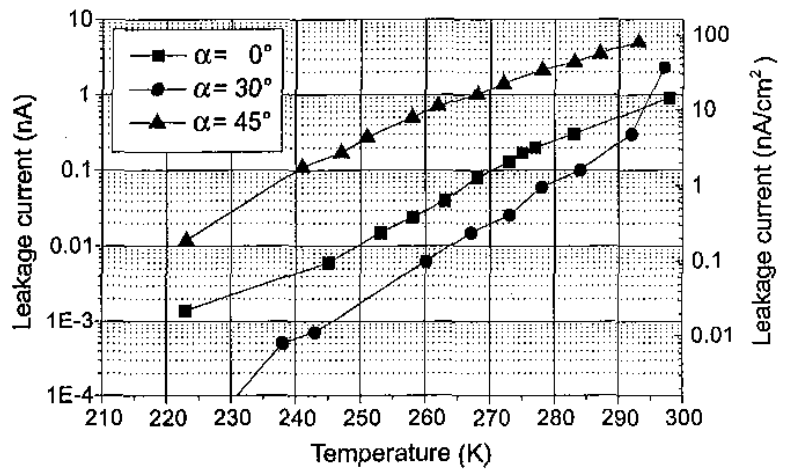

Figure 4: Leakage current as a function of temperature for MSSDD with different $a$ at the operating voltage.

\section{Operation Stability}

The biasing of our large linear SDD leads to a high-power consumption in the resistor chains heating up the detector. Consequently, leakage currents collected at anode pixels continuously increase. The achievable energy resolution worsens and is time dependent. To minimize this, design of internal resistor chain and experimental arrangement of external resistors has to be done carefully. For the tested detector, we had to supply the external and internal resistor chains with a total current of $\sim 5.34 \mathrm{~mA}(\sim 4.6 \mathrm{~mA}$ in the external and $\sim 0.74 \mathrm{~mA}$ in the internal resistor chain) in order to establish the desired potential distribution. According to the total voltage drop along the resistor chains, the power dissipation is $\sim 1.84 \mathrm{~W}$ and $\sim 0.21 \mathrm{~W}$ in the external and internal resistor chains, respectively. We have measured that this power dissipation increases the temperature of the detector with a rate of $\sim 0.3 \mathrm{~K} / \mathrm{min}$. even if the nitrogen cooling was active. The temperature probe was placed on the box with the detector. We expect that the temperature will be locally much higher inside the detector itself than indicated by the probe. 


\section{X-ray Spectroscopy at Room Temperature}

The ${ }^{241} \mathrm{Am}$ spectra collected with an MSSDD for $\alpha=0^{\circ}$ and $30^{\circ}$ at room temperature are shown in Fig. 5a-b. In the case of free diffusion $\left(\alpha=0^{\circ}\right)$, we see a significant worsening of the spectroscopic quality (see Fig. 5a) characterized by a large lowenergy tail due to the high amount of split events and a shift of the peaks to lower energies. Moreover the peak corresponding to $59.54 \mathrm{keV} \gamma$-rays is completely lost. We have calculated the ${ }^{241} \mathrm{Am}$ spectrum (see inset in Fig. 5a) using the applied drift field and a realistic estimation of the irradiated area [4]. The best fit between the calculated spectrum and the measured one is achieved for FWHM $=0.8 \mathrm{keV}$ ( $92 \mathrm{rms}$ electrons) measured at the $13.9 \mathrm{keV}$ line. In the case of $\alpha=30^{\circ}$, an improvement of the spectroscopic quality of the collected spectrum (see Fig. $5 b$ ) due to elimination of diffusion is clearly visible. The peaks can be well fitted with a gaussian function with a FWHM $=1.4$ $\mathrm{keV}$ (164 rms electrons).
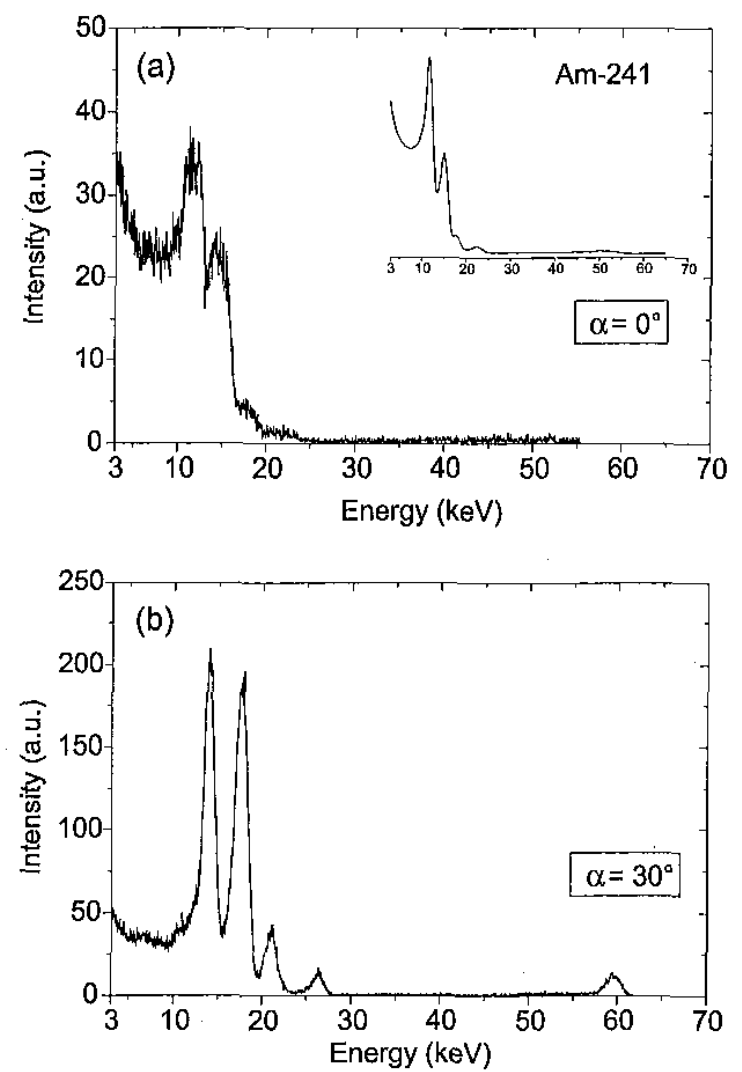

Figure 5: Pulse height spectra of ${ }^{241}$ Am measured with one anode pixel of MSSDD for $\alpha=0^{\circ}$ (a) and $\alpha=30^{\circ}(\mathrm{b})$. The measurements were done at room temperature $(\mathrm{T}=293 \mathrm{~K})$ with a shaping time of 0.5 $\mu \mathrm{s}$. The inset in (a) shows the calculated spectrum.

\section{E. $X$-ray Spectroscopy at Low Temperature}

${ }^{55} \mathrm{Fe}$ spectra obtained with an MSSDD for $\alpha=0^{\circ}$ and $30^{\circ}$ at low temperature are shown in Fig. 6a-b. Although we have measured a large drop of the leakage current at low temperature (see Sect. III.B), the best energy resolution is obtained with a shaping time of $1 \mu \mathrm{s}$ and $0.5 \mu \mathrm{s}$ for $\alpha=0^{\circ}$ and $30^{\circ}$, respectively. We have measured a FWHM $=260 \mathrm{eV}(28 \mathrm{rms}$ electrons, fitted with a diffusion-based model) and $450 \mathrm{eV}(52 \mathrm{rms}$ electrons, fitted with a gaussian function) for $\alpha=0^{\circ}$ and $30^{\circ}$, respectively.
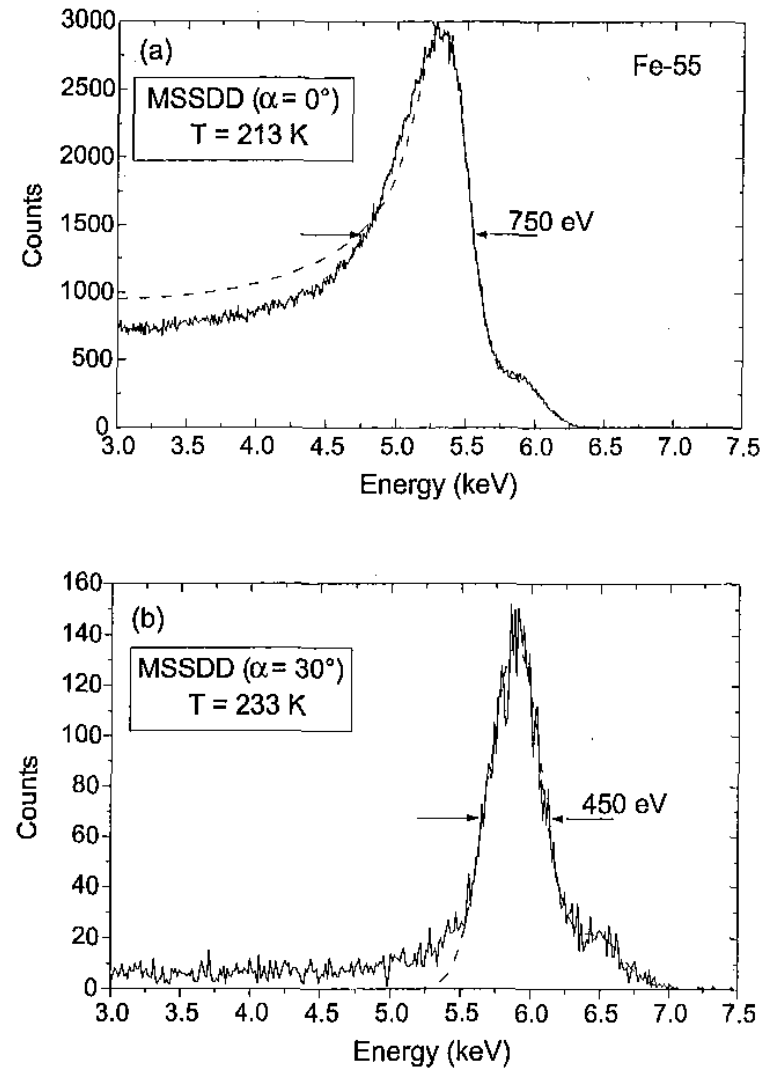

Figure 6: Pulse height spectra of ${ }^{55} \mathrm{Fe}$ measured with one anode pixel of MSSDD for $\alpha=0^{\circ}$ (a) and $\alpha=30^{\circ}$ (b) at low temperature. The dashed lines show the best fitting using diffusion-based model (a) and a gaussian function (b). The measured $\mathrm{FWHM}_{\text {total }}$ are indicated.

\section{Discussion AND CONCLUSION}

The summary of the presented measurements with an MSSDD is reported in Table 1. The split events due to diffusion (an MSSDD with $\alpha=0^{\circ}$ ) result in a large low-energy tail (see FWHM $_{\text {split }}$ in Table 1) and a high background (indicated by $\mathrm{R}_{\text {peak }}$ in Table 1). The collected spectrum can be well fitted with a simple model assuming the influence of diffusion and the noise contribution of the detector to the FWHM total $_{\text {can }}$ be extracted. The split cvents due to diffusion are eliminated by using sawtooth shaped $\mathrm{p}^{+}$strips (an MSSDD with $\alpha=30^{\circ}$ ). In this case, the additional low-energy broadening of observed pcaks is completely eliminated $\left(\mathrm{FWHM}_{s p l i t}=0 \mathrm{eV}\right)$ and only a relatively small background is observed. We assume that this is due to doping inhomogeneities and edge effects. To analyzc the impact of the edge effect, an ${ }^{55} \mathrm{Fe}$ spectrum was collected with a relatively small circular SDD with an active area of only $0.71 \mathrm{~mm}^{2}$ (see Table 1). The edge effects of this small circular SDD will be similar to those of our MSSDD. The values of 
Table 1

Comparison of spectroscopic quality of MSSDD with $\alpha=0^{\circ}$ and $30^{\circ}$ obtained from measured ${ }^{55} \mathrm{Fe}$ spectra

\begin{tabular}{|c|c|c|c|c|c|c|c|}
\hline & $\mathrm{T}(\mathrm{K})$ & $\begin{array}{c}\text { shaping } \\
\text { time }(\mu \mathrm{s})\end{array}$ & $\begin{array}{c}\text { FWHM } \\
(\mathrm{keV})\end{array}$ & $\begin{array}{c}\mathrm{FWHM}_{\text {spit }} \\
(\mathrm{keV})\end{array}$ & $\begin{array}{c}\text { ENC } \\
\left(\mathrm{e}^{-} \mathrm{rms}\right)\end{array}$ & $\mathrm{R}_{\text {peak }}$ & $\mathrm{R}_{\text {count }}$ \\
\hline \multirow{2}{*}{ MSSDD $\left(\alpha=0^{\circ}\right)$} & 293 & 0.5 & 1.6 & 1.4 & $92^{a}$ & 2.6 & $-^{c}$ \\
\cline { 2 - 8 } & 213 & 1.0 & 0.75 & 0.7 & $28^{a}$ & 4 & $\bar{c}^{c}$ \\
\hline \multirow{2}{*}{ MSSDD $\left(\alpha=30^{\circ}\right)$} & 293 & 0.5 & 1.4 & 0 & $164^{b}$ & 8 & 2.8 \\
\cline { 2 - 8 } & 233 & 0.5 & 0.45 & 0 & $52^{b}$ & 25 & 2.3 \\
\hline $\begin{array}{c}\text { Circular SDD } \\
\left(0.71 \mathrm{~mm}^{2}\right)\end{array}$ & 293 & 0.5 & 0.36 & 0 & $40^{b}$ & 40 & 2.8 \\
\hline
\end{tabular}

${ }^{a}$ Fitted by diffision-based model [4].

${ }^{b}$ Fitted by a gaussian function.

${ }^{c}$ Only split events are collected, because the more distant part of the active area of the tested anode pixel is irradiated (in fact $R_{\text {count }}=0$ ) [4].

$\mathrm{R}_{\text {count }}$, the parameter describing the background, are matching well for both detectors.

The energy resolution of an MSSDD at room temperature due to electronic noise is mainly determined by the relatively high leakage current. Knowing the noise parameters of the JFET and the leakage current of the detector, the total electronic noise can be calculated as a function of the shaping time and temperature [12]. We have calculated that $\mathrm{ENC}=$ 80 and $113 \mathrm{rms}$ electrons for an MSSDD with $\alpha=0^{\circ}$ and $30^{\circ}$, respectively, at room temperature and shaping time of 0.5 $\mu \mathrm{s}$. At low temperature, leakage current drastically drops and the $1 / f$ noise and the total input capacitance are expected to be the main contributions to the electronic noise. The noise observed in ${ }^{55} \mathrm{Fe}$ spectra is still the lowest for a short shaping time of $\leq 1 \mu \mathrm{s}$ (see Fig. 6a-b), although we expected that the lowest noise would be achieved for a shaping time of 3-6 $\mu \mathrm{s}$. We have calculated that $\mathrm{ENC}=25$ and $30 \mathrm{rms}$ electrons for an MSSDD with $\alpha=0^{\circ}(@ \mathrm{~T}=213 \mathrm{~K}$ and $\tau=1 \mu \mathrm{s})$ and $30^{\circ}$ (@) $\mathrm{T}=233 \mathrm{~K}$ and $\tau=0.5 \mu \mathrm{s}$ ), respectively. The rms noise values obtained from the measured ${ }^{55} \mathrm{Fe}$ spectra (see Table 1) match those obtained by calculation using the measured leakage current of the detector and the JFET noise parameters reasonably well. In general, the calculated rms noise values are smaller, because the leakage current used for the calculation is measured immediately after biasing the detector. In this manner, the increase of the leakage current due to an increase of the temperature (see Sect. III.C) is not taken into account.

In conclusion, X-ray spectroscopy measurements clearly showed the elimination of diffusion in a MSSDD with $\alpha=$ $30^{\circ}$. At low temperatures, the energy resolution is dominated by the $1 / \mathrm{f}$ noise, the total input capacitance and the heating of our MSSDD at long-time scale. The results of X-ray spectroscopy measurements are reported for $\alpha=30^{\circ}$. Similar sets of measurements were also carried out for $\alpha=45^{\circ}$ and $60^{\circ}$. The obtained results are identical besides the differences in electronic noise due to different leakage currents.

Future plans include integration of the front-end JFET and the improvement of the detector design, especially the design of the anode region and the internal resistor chains to reduce leakage current. The experimental arrangement will also be improved.

\section{REFERENCES}

[1] L. Strüder et al., "High Resolution, High Speed Detectors with Integrated JFET Electronics," J. Phys, IV, vol. 7(suppl. C2), 1997 pp. 21-29.

[2] R. Hartmann, L. Strüder, J. Kemmer, P. Lechner, O. Fries, E. Lorenz, and R. Mirzoyan, "Ultrathin entrance windows for silicon drift detectors," Nucl. Instrum. Methods, vol. A387, 1997 pp. 250-254.

[3] E. Gatti, A. Longoni, P. Rehak, and M. Sampietro, "Dynamics of electrons in drift detectors," Nucl. Instrum. Methods, vol. A 253, 1987 pp. 393-399.

[4] J. Šonský, R.W. Hollander, P.M. Sarro, and C.W.E. van Eijk, "X-ray spectroscopy with a Multi-anode Sawtooth Silicon Drift Detector: the diffusion process," submitted for publication to Nucl. Instrum. Methods A.

[5] E.A. Hijzen, E.M. Schooneveld, C.W.E. van Eijk, and R.W. Hollander, "New silicon drift detector design for diminishing lateral diffusion," Nucl. Instrum. Methods, vol. $A 335,1993$ pp. $271-275$.

[6] J. Šonský, H. Valk, C.P. Allier, R.W. Hollander, C.W.E. van Eijk, and P.M. Sarro, "Diminished Electron Cloud Broadening in a Silicon Drift Detector by Saw Tooth $\mathrm{p}^{+}$ Strips," IEEE Trans. Nucl. Sci., vol. 46, 1999 pp. 53--58.

[7] J. Šnský, H. Valk, J. Huizenga, R.W. Hollander, C.W.E. van Eijk, and P.M. Sarro, "Electron Confinement in MultiAnode Saw Tooth Silicon Drift Detectors With an Anode Pitch of $250 \mu \mathrm{m}$," IEEE Trans. Nucl. Sci, vol. 46, 1999 pp. 271-274.

[8] G. Bertuccio, P. Rehak, and D. Xi, "A novel charge sensitive preamplifier without the feedback resistor," $\mathrm{Nucl}$. Instrum. Methods, vol. A326, 1993 pp. 71-76.

[9] L.K. Nanver, E.J.G. Goudena, and H.W. van Zeijl, "Optimization of fully-implanted NPN's for high- 
frequency operation," IEEE Trans. on Electron Devices, vol. 43(6), $1996 \mathrm{pp}, 1038-1040$.

[10] J.R. Hall et al., "Star Silicon Drift Detectors - Detector Tests," STAR Note \#171, 1994 internal report.

[11] C.P. Allier, H. Valk, J. Huizenga, V.R. Bom, R.W. Hollander, and C.W.E. van Eijk, "Comparative Study of Silicon Detectors," IEEE Trans. Nucl. Sci., vol. 45, 1998 pp. 576-580.

[12] G. Bertuccio and A. Pullia, "A method for the determination of the noise parameters in preamplifying systems for semiconductor radiation detectors," Rev. Sci. Instrum., vol. 64(11), 1993 pp. 3294-3298. 